IJMMS 31:11 (2002) 687-694

PII. S0161171202110027

http://ijmms.hindawi.com

(c) Hindawi Publishing Corp.

\title{
SOLVING THE DIRICHLET ACOUSTIC SCATTERING PROBLEM FOR A SURFACE WITH ADDED BUMPS USING THE GREEN'S FUNCTION FOR THE ORIGINAL SURFACE
}

\author{
MAXIM J. GOLDBERG and SEONJA KIM
}

Received 1 October 2001

\begin{abstract}
We solve the Dirichlet problem for acoustic scattering from a surface which has been perturbed by the addition of one or more bumps. We build the solution for the bumpy case using the Green's function for the unperturbed surface, and the solution of a local integral equation in which the integration is carried out only over the added bumps. We conclude by giving an alternative formulation of our method for the special case of a bump on a plane.
\end{abstract}

2000 Mathematics Subject Classification: 35J25, 35J05.

1. Introduction. In many applications, it is desirable to study scattering from a surface to which extra features have been "added," for example, growths sticking out of background tissue, some objects appearing in the middle of a field, and so forth. In this paper, we assume that we know Green's function for the original surface, or, what is equivalent, that we can solve the Dirichlet problem for the original surface. Using this information, and given the boundary values of the field on the perturbed surface, we reach an integral equation of the second kind in which the integration is carried out only over the added bumps, not over the entire perturbed surface. We use the solution of the latter equation, the given boundary values, and the derivative of the Green's function for the unperturbed surface to solve the Dirichlet acoustic scattering problem for the perturbed surface. We conclude by giving an alternative formulation of our method for the special case of a bump on a plane.

In what follows, the wavenumber $k$ is fixed. By solving the Dirichlet acoustic scattering problem for a surface we aim at finding scalar function $u$ which (1) satisfies the Helmholtz equation $\Delta u+k^{2} u=0$ in the exterior of the surface, (2) has prescribed boundary values on the surface, and (3) satisfies the Sommerfeld radiation condition at infinity (see [2] for more details). A function satisfying the Helmholtz equation will be called metaharmonic.

We will assume that all surfaces and functions involved are as smooth as necessary to ensure that all formulas that we use are valid, and that pointwise limits exist almost everywhere; in particular, Lipschitz surfaces are allowed. Limits will be understood to hold almost everywhere.

2. Derivation of the solution. Let $\partial \Omega^{0}$ denote the original surface. We write

$$
\partial \Omega^{0}=A \cup B^{0},
$$


where $A$ and $B^{0}$ are partitions of the surface, and $B^{0}$ is the part of the surface above which the bumps are added. Let $B$ denote these bumps, and let

$$
\partial \Omega=A \cup B
$$

denote the perturbed surface. We suppose that $B$ lies above $B^{0}$. For $X, Y$ outside $\partial \Omega^{0}$, let $G^{0}(X, Y)$ denote the Green's function associated with the operator $\Delta+k^{2}$ (where $k$ is the wavenumber) for the exterior of $\partial \Omega^{0}$. Note that

$$
G^{0}(X, Y)=\Phi(X, Y)-U^{0}(X, Y)
$$

where $\Phi(X, Y)$ is the free space Green's function

$$
\frac{e^{i k|X-Y|}}{4 \pi|X-Y|}
$$

and $U^{0}(X, Y)$, considered as a function of $Y$, is the metaharmonic extension above $\partial \Omega^{0}$ of $\left.\Phi(X, Y)\right|_{Y \in \partial \Omega^{0}} ; U^{0}$ also satisfies the Sommerfeld radiation condition. Thus, being able to solve the Dirichlet problem for a surface is equivalent to knowing the Green's function for all pairs of points in the exterior of the surface (see also Note 2.3). We can actually take $X$ or $Y$ to be on $\partial \Omega^{0}$.

Note 2.1. It is well known that $G^{0}(X, Y)=G^{0}(Y, X)$. This can be shown by using Green's formula on the exterior of $\partial \Omega^{0}$ for the function

$$
R(Z)=G^{0}(X, Z) \frac{\partial G^{0}(Y, Z)}{\partial n(Z)}-G^{0}(Y, Z) \frac{\partial G^{0}(X, Z)}{\partial n(Z)}
$$

excluding two balls of radius $\epsilon$ centered at $X$ and $Y$, respectively, and then letting $\epsilon \rightarrow 0$ (see also [3]).

Note 2.2. For $X$ in the exterior of $\partial \Omega^{0}$,

$$
\left.G^{0}(X, Y)\right|_{Y \in \partial \Omega^{0}}=0
$$

Note 2.3. For a function $h$ given on $\partial \Omega^{0}$,

$$
h(Z)=\lim _{X \rightarrow Z} \int_{\partial \Omega^{0}} h(Y) \frac{\partial G^{0}(X, Y)}{\partial n(Y)} d \sigma(Y),
$$

for $Z \in \partial \Omega^{0}$, and where $X$ stays in the exterior of $\partial \Omega$ and converges to $Z$ nontangentially. 
Proof. Let $H$ be the metaharmonic extension of $h$ outside $\partial \Omega^{0}$, namely, for $X$ outside $\partial \Omega^{0}$,

$$
H(X)=\int_{\partial \Omega^{0}}\left(h(Y) \frac{\partial \Phi(X, Y)}{\partial n(Y)}-\frac{\partial h(Y)}{\partial n(Y)} \Phi(X, Y)\right) d \sigma(Y)
$$

The above formula gives the unique metaharmonic extension satisfying the Sommerfeld radiation condition at infinity (see [2]). We then use Green's formula again to interchange the derivative in the unit normal direction in the second term of the formula above, noting that the derivative of the metaharmonic extension of $\Phi$ outside the boundary should be used instead of the derivative of $\Phi$ itself. We obtain

$$
\begin{aligned}
H(X) & =\int_{\partial \Omega^{0}} h(Y) \frac{\partial}{\partial n(Y)}\left(\Phi(X, Y)-U^{0}(X, Y)\right) d \sigma(Y) \\
& =\int_{\partial \Omega^{0}} h(Y) \frac{\partial G^{0}(X, Y)}{\partial n(Y)} d \sigma(Y)
\end{aligned}
$$

where we have used (2.3) for the second equality. Sending $X \rightarrow Z$ we obtain the desired result.

Now, let $g$ be the given boundary values on the perturbed surface $\partial \Omega$. Our goal is to construct a solution for the Helmholtz equation in the exterior of $\partial \Omega$ with boundary values $g$, which also satisfies the Sommerfeld radiation condition, using $G^{0}(X, Y)$, Green's function for the unperturbed surface, and solving an integral equation in which the integration is done only over the bump region $B$ in $\partial \Omega$.

For $X$ in the exterior of $\partial \Omega$, define

$$
Q(f)(X)=\int_{\partial \Omega} f(Y) \frac{\partial G^{0}(X, Y)}{\partial n(Y)} d \sigma(Y)
$$

Clearly, $Q(f)$ is metaharmonic in the exterior of $\partial \Omega$. We want to find a function $f$ so that, for (almost) every $Z \in \partial \Omega$,

$$
g(Z)=\lim _{X \rightarrow Z} Q(f)(X)
$$

as $X$ approaches $Z$ nontangentially in the exterior of $\partial \Omega$. We will never consider what happens at $Z$ 's which belong to (boundary $A$ ) $\cup$ (boundary $B$ ), a set assumed to be of measure 0 .

Now, let $Z$ be a point in $A$, the set common to both $\partial \Omega$ and $\partial \Omega^{0}$. We send $X \rightarrow Z$ nontangentially in (2.10). In the derivation below, we use the function

$$
h(Y)= \begin{cases}f(Y), & \text { for } Y \in A, \\ 0, & \text { for } Y \in B^{0}\end{cases}
$$


where $B^{0}$ is the part of $\partial \Omega^{0}$ below the bumps $B$ in $\partial \Omega$

$$
\begin{aligned}
\lim _{X \rightarrow Z} Q(f)(X)= & \lim _{X \rightarrow Z} \int_{\partial \Omega} f(Y) \frac{\partial G^{0}(X, Y)}{\partial n(Y)} d \sigma(Y) \\
= & \lim _{X \rightarrow Z} \int_{A} f(Y) \frac{\partial G^{0}(X, Y)}{\partial n(Y)} d \sigma(Y) \\
& +\lim _{X \rightarrow Z} \int_{B} f(Y) \frac{\partial G^{0}(X, Y)}{\partial n(Y)} d \sigma(Y) \\
= & \lim _{X \rightarrow Z} \int_{\partial \Omega^{0}} h(Y) \frac{\partial G^{0}(X, Y)}{\partial n(Y)} d \sigma(Y) \\
& +\lim _{X \rightarrow Z} \int_{B} f(Y) \frac{\partial G^{0}(X, Y)}{\partial n(Y)} d \sigma(Y) \\
= & f(Z)+0 \\
= & f(Z) .
\end{aligned}
$$

We have used Note 2.3 in the above derivation and the fact that $h(Z)=f(Z)$, for $Z \in A$. It can be easily seen that

$$
\lim _{X \rightarrow Z} \int_{B} f(Y) \frac{\partial G^{0}(X, Y)}{\partial n(Y)} d \sigma(Y)=0
$$

as follows. Consider the bounded region $B \cup B^{0}$. Let

$$
p(Y)= \begin{cases}f(Y), & \text { for } Y \in B, \\ 0, & \text { for } Y \in B^{0}\end{cases}
$$

and denote by $P$ a metaharmonic extension of $p$ inside $B \cup B^{0}$. Next, note that

$$
\begin{array}{rl}
\lim _{X \rightarrow Z} \int_{B} & f(Y) \frac{\partial G^{0}(X, Y)}{\partial n(Y)} d \sigma(Y) \\
& =\lim _{X \rightarrow Z} \int_{B \cup B^{0}} p(Y) \frac{\partial G^{0}(X, Y)}{\partial n(Y)} d \sigma(Y) \\
& =\lim _{X \rightarrow Z} \int_{B \cup B^{0}}\left(P(Y) \frac{\partial G^{0}(X, Y)}{\partial n(Y)}-G^{0}(X, Y) \frac{\partial P(Y)}{\partial n(Y)}\right) d \sigma(Y),
\end{array}
$$

since

$$
\lim _{X \rightarrow Z} G^{0}(X, Y)=\lim _{X \rightarrow Z} G^{0}(Y, X)=0 .
$$

Now use Green's theorem for the interior of $B \cup B^{0}$ to conclude that the last line in (2.16) equals zero. Thus, we take $f(Y)=g(Y)$ for $Y \in A$. 
Now, let $Z$ be in $B$ and send $X \rightarrow Z$ nontangentially in (2.10). We obtain

$$
\begin{aligned}
\lim _{X \rightarrow Z} Q(f)(X)= & \lim _{X \rightarrow Z} \int_{\partial \Omega} f(Y) \frac{\partial G^{0}(X, Y)}{\partial n(Y)} d \sigma(Y) \\
= & \lim _{X \rightarrow Z} \int_{\partial \Omega} f(Y) \frac{\partial \Phi(X, Y)}{\partial n(Y)} d \sigma(Y) \\
& -\lim _{X \rightarrow Z} \int_{\partial \Omega} f(Y) \frac{\partial U^{0}(X, Y)}{\partial n(Y)} d \sigma(Y) \\
= & \frac{1}{2} f(Z)+\int_{\partial \Omega} f(Y) \frac{\partial \Phi(Z, Y)}{\partial n(Y)} d \sigma(Y) \\
& -\int_{\partial \Omega} f(Y) \frac{\partial U^{0}(Z, Y)}{\partial n(Y)} d \sigma(Y) \\
= & \frac{1}{2} f(Z)+\int_{\partial \Omega} f(Y) \frac{\partial G^{0}(Z, Y)}{\partial n(Y)} d \sigma(Y) .
\end{aligned}
$$

Here, we used the properties of limits of double layer potentials, see [2], and that $U^{0}(X, Y)$ is nonsingular for $X$ above $\partial \Omega^{0}$. So, to have (2.11) hold, we need to solve

$$
\begin{aligned}
g(Z)= & \frac{1}{2} f(Z)+\int_{A} f(Y) \frac{\partial G^{0}(Z, Y)}{\partial n(Y)} d \sigma(Y) \\
& +\int_{B} f(Y) \frac{\partial G^{0}(Z, Y)}{\partial n(Y)} d \sigma(Y),
\end{aligned}
$$

for $Z \in B$. But we are taking $f(Y)=g(Y)$ for $Y \in A$, so we want to find $f$ so that

$$
\frac{1}{2} f(Z)+\int_{B} f(Y) \frac{\partial G^{0}(Z, Y)}{\partial n(Y)} d \sigma(Y)=g(Z)-\int_{A} g(Y) \frac{\partial G^{0}(Z, Y)}{\partial n(Y)} d \sigma(Y),
$$

for $Z \in B$.

Thus, the solution to the Dirichlet problem for $\partial \Omega$ can be obtained as follows. Given $g$ on $\partial \Omega$, define a function $h$ on $\partial \Omega^{0}$ by

$$
h(Y)= \begin{cases}g(Y), & \text { for } Y \in A, \\ 0, & \text { for } Y \in B^{0} .\end{cases}
$$

For $X$ in the exterior of $\partial \Omega^{0}$, define the function $H$ by

$$
H(X)=\int_{\partial \Omega^{0}} h(Y) \frac{\partial G^{0}(X, Y)}{\partial n(Y)} d \sigma(Y) .
$$

Next, solve the following integral equation:

$$
\frac{1}{2} f(Z)+\int_{B} f(Y) \frac{\partial G^{0}(Z, Y)}{\partial n(Y)} d \sigma(Y)=g(Z)-H(Z),
$$

for $Z \in B$. Define $\tilde{f}$ by

$$
\tilde{f}= \begin{cases}g(Y), & \text { for } Y \in A, \\ f(Y), & \text { for } Y \in B\end{cases}
$$


Then (2.13) and (2.18), and the discussions following them, show that for almost every $Z \in \partial \Omega$,

$$
Q(\tilde{f})(X)=\int_{\partial \Omega} \tilde{f}(Y) \frac{\partial G^{0}(X, Y)}{\partial n(Y)} d \sigma(Y) \rightarrow g(Z),
$$

as $X$ in the exterior of $\partial \Omega$ converges nontangentially to $Z$. Hence, $Q(\tilde{f})$ solves the Dirichlet acoustic scattering problem for the given boundary values $g$ on $\partial \Omega$.

3. Special case. We apply the above recipe to the case when $\partial \Omega^{0}$ is the $x y$ plane, and for simplicity $B$ is just one bump on the plane; the case of several bumps on the plane is similar. Then

$$
G^{0}(X, Y)=\Phi(X, Y)-\Phi\left(X, Y^{*}\right)
$$

where, if $Y=\left(y_{1}, y_{2}, y_{3}\right), Y^{*}=\left(y_{1}, y_{2},-y_{3}\right)$. Let

$$
h(Y)= \begin{cases}g(Y), & \text { for } Y=\left(y_{1}, y_{2}, 0\right) \in A, \\ 0, & \text { for } Y \in B^{0}\end{cases}
$$

Then

$$
H(X)=\int_{\partial \Omega^{0}} h(Y) \frac{\partial G^{0}(X, Y)}{\partial n(Y)} d \sigma(Y)
$$

is the unique metaharmonic extension of $h$ to the region above the plane (which also satisfies the radiation condition).

For $Z \in B$, our procedure in Section 2 calls to solve

$$
\frac{1}{2} f(Z)+\int_{B} f(Y) \frac{\partial G^{0}(Z, Y)}{\partial n(Y)} d \sigma(Y)=g(Z)-H(Z) .
$$

Now, let $B^{*}$ be the reflection of $B$ about the $x y$ plane. In other words, $Y \in B$ if and only if $Y^{*} \in B^{*}$, where $Y^{*}$ has been defined in (3.1). Note that $B \cup B^{*}$ fully encloses a region. Also, if $n(Y)$ denotes the outward unit normal at $Y \in B$, and $n\left(Y^{*}\right)$ denotes the outward unit normal at $Y^{*} \in B^{*}$, we have

$$
n\left(Y^{*}\right)=[n(Y)]^{*} .
$$

So, for $Y \in B$ (and $Z \in B$ ),

$$
\begin{aligned}
\frac{\partial}{\partial n(Y)}\left(\Phi\left(Z, Y^{*}\right)\right) & =\left.\left.\left(n_{1}, n_{2}, n_{3}\right)\right|_{Y} \cdot\left(D_{1} \Phi, D_{2} \Phi,-D_{3} \Phi\right)\right|_{Y^{*}} \\
& =\left.\left.\left(n_{1}, n_{2},-n_{3}\right)\right|_{Y} \cdot\left(D_{1} \Phi, D_{2} \Phi, D_{3} \Phi\right)\right|_{Y^{*}} \\
& =\left.\left.\left(n_{1}, n_{2}, n_{3}\right)\right|_{Y^{*}} \cdot\left(D_{1} \Phi, D_{2} \Phi, D_{3} \Phi\right)\right|_{Y^{*}} \\
& =\frac{\partial \Phi\left(Z, Y^{*}\right)}{\partial n\left(Y^{*}\right)} .
\end{aligned}
$$


Hence, we find that

$$
\int_{B} f(Y) \frac{\partial}{\partial n(Y)}\left(\Phi\left(Z, Y^{*}\right)\right) d \sigma(Y)=\int_{B^{*}} f\left(Y^{*}\right) \frac{\partial \Phi(Z, Y)}{\partial n(Y)} d \sigma(Y) .
$$

Thus,

$$
\begin{aligned}
\int_{B} f(Y) \frac{\partial G^{0}(Z, Y)}{\partial n(Y)} d \sigma(Y) \\
\quad=\int_{B} f(Y) \frac{\partial \Phi(Z, Y)}{\partial n(Y)} d \sigma(Y)-\int_{B} f(Y) \frac{\partial}{\partial n(Y)}\left(\Phi\left(Z, Y^{*}\right)\right) d \sigma(Y) \\
\quad=\int_{B \cup B^{*}} \tilde{f}(Y) \frac{\partial \Phi(Z, Y)}{\partial n(Y)} d \sigma(Y)
\end{aligned}
$$

where

$$
\tilde{f}(Y)= \begin{cases}f(Y), & \text { for } Y \in B \\ -f\left(Y^{*}\right), & \text { for } Y \in B^{*}\end{cases}
$$

So (3.4) becomes

$$
\frac{1}{2} \tilde{f}(Z)+\int_{B \cup B^{*}} \tilde{f}(Y) \frac{\partial \Phi(Z, Y)}{\partial n(Y)} d \sigma(Y)=g(Z)-H(Z),
$$

for $Z \in B$. Substituting $Z^{*}$ for $Z$ in (3.10), it is easy to see that (3.10) is equivalent to

$$
\frac{1}{2} \tilde{f}(Z)+\int_{B \cup B^{*}} \tilde{f}(Y) \frac{\partial \Phi(Z, Y)}{\partial n(Y)} d \sigma(Y)=-\left(g\left(Z^{*}\right)-H\left(Z^{*}\right)\right),
$$

for $Z \in B^{*}$. Thus, solving (3.10) or (3.11) is equivalent to solving for $p$ in

$$
\frac{1}{2} p(Z)+\int_{B \cup B^{*}} p(Y) \frac{\partial \Phi(Z, Y)}{\partial n(Y)} d \sigma(Y)=R(Z),
$$

for $Z \in B \cup B^{*}$, and where

$$
R(Z)= \begin{cases}g(Z)-H(Z), & \text { for } Z \in B \\ -\left(g\left(Z^{*}\right)-H\left(Z^{*}\right)\right), & \text { for } Z \in B^{*}\end{cases}
$$

Note that (3.12) arises when using the double layer potential method for finding the metaharmonic extension, which satisfies the Sommerfeld radiation condition, outside the symmetric region bounded by $B \cup B^{*}$ with the boundary values on the lower half of the surface $B \cup B^{*}$ being the negatives of the boundary values at symmetric points on the upper half of the surface. Also note, that the solution $p$ will be necessarily antisymmetric on $B \cup B^{*}$, that is, $p(Z)=-p\left(Z^{*}\right)$, as we need.

Finally, it is easy to check that

$$
H(X)+\int_{B \cup B^{*}} p(Y) \frac{\partial \Phi(X, Y)}{\partial n(Y)} d \sigma(Y) \rightarrow g(Z),
$$


as $X$ above $\partial \Omega$ approaches $Z$ nontangentially. The idea of solving the Dirichlet problem for a plane with a bump, by adding the solution for the plane without the bump to the solution for the symmetrized bump (with antisymmetric boundary values), has been used earlier in [1] to obtain comparison values.

\section{REFERENCES}

[1] R. Coifman, M. Goldberg, T. Hrycak, M. Israeli, and V. Rokhlin, An improved operator expansion algorithm for direct and inverse scattering computations, Waves Random Media 9 (1999), no. 3, 441-457.

[2] D. L. Colton and R. Kress, Integral Equation Methods in Scattering Theory, Pure and Applied Mathematics, John Wiley \& Sons, New York, 1983.

[3] G. F. Roach, Green's Functions, 2nd ed., Cambridge University Press, Cambridge, 1982.

Maxim J. Goldberg: Physical Sciences Department, York College of Pennsylvania, YORK, PA 17405, USA

Current address: School of TheORETiCAl AND APPlied SCIENCE, RAMAPo College OF NeW JERSEY, 505 RAMAPO VALLEY ROAD, MAHWAH, NJ 07430, USA

E-mail address: mgoldberg@ramapo.edu

SeONJA Kim: School of Computer SCIENCE AND Information Systems, FAIRLeigh DickINSON UNIVERSITY, 1000 RIVER ROAD, MAIL CODE T-BE2-01, TEANECK, NJ 07666, USA

E-mail address: skim@fdu.edu 


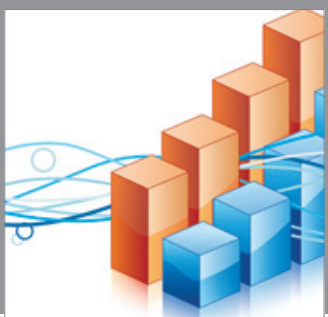

Advances in

Operations Research

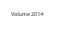

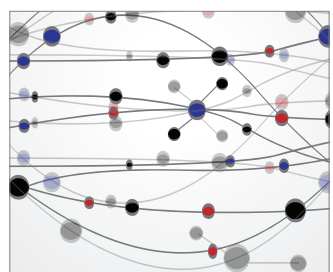

\section{The Scientific} World Journal
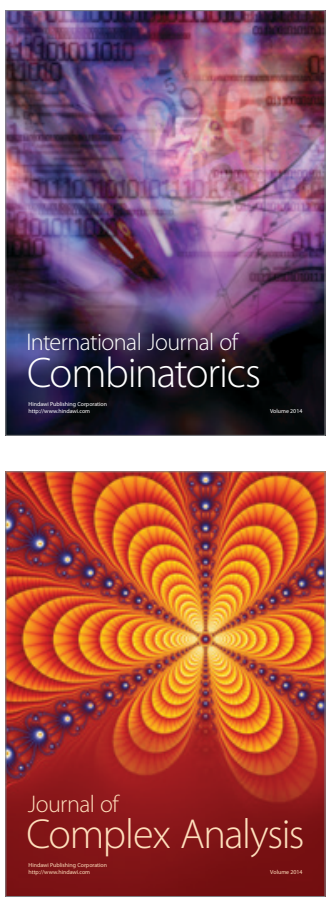

International Journal of

Mathematics and

Mathematical

Sciences
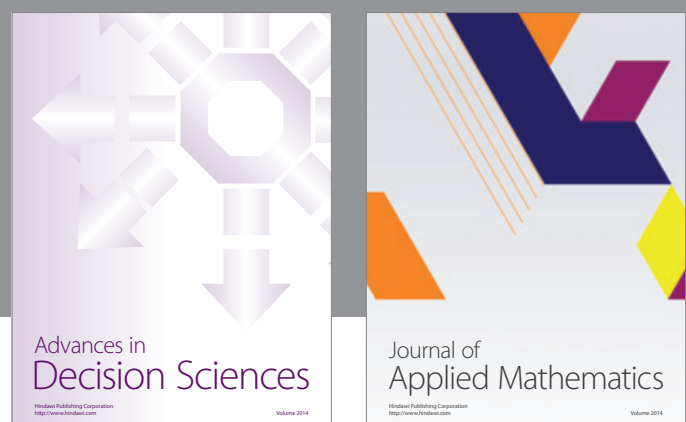

Journal of

Applied Mathematics
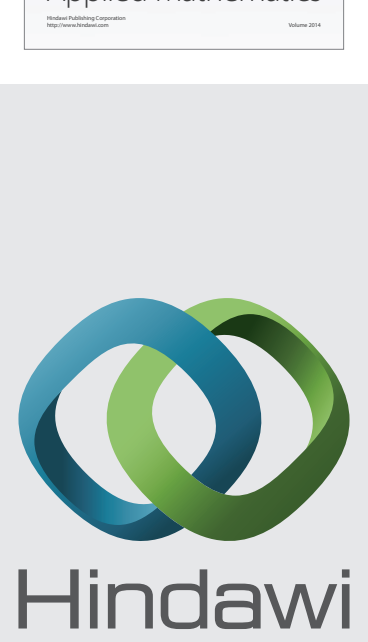

Submit your manuscripts at http://www.hindawi.com
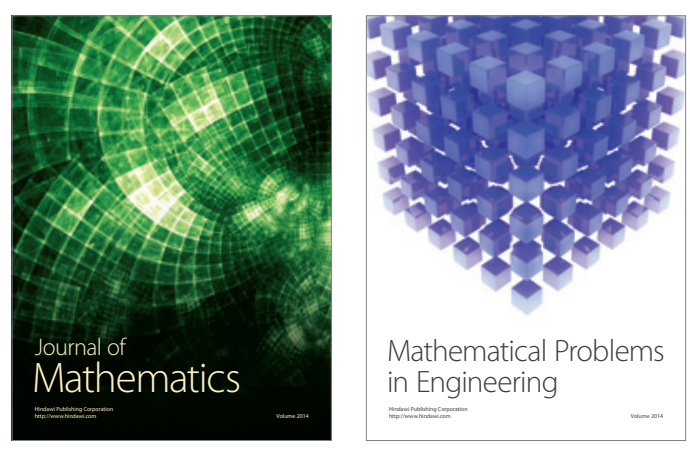

Mathematical Problems in Engineering
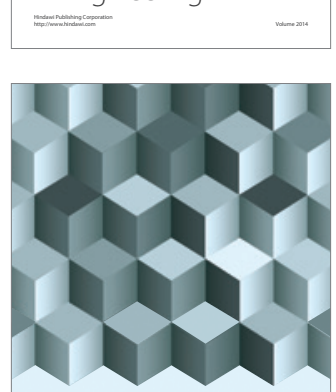

Journal of

Function Spaces
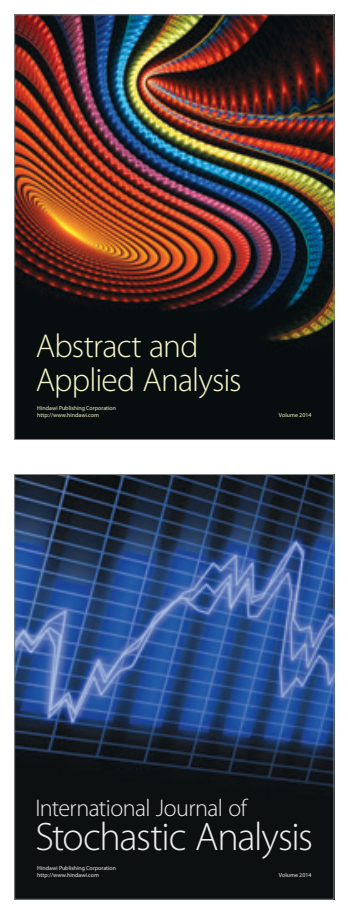

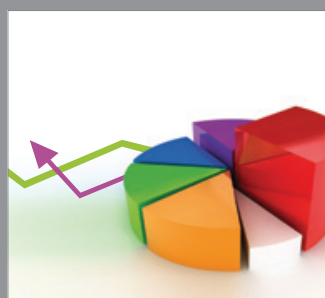

ournal of

Probability and Statistics

Promensencen
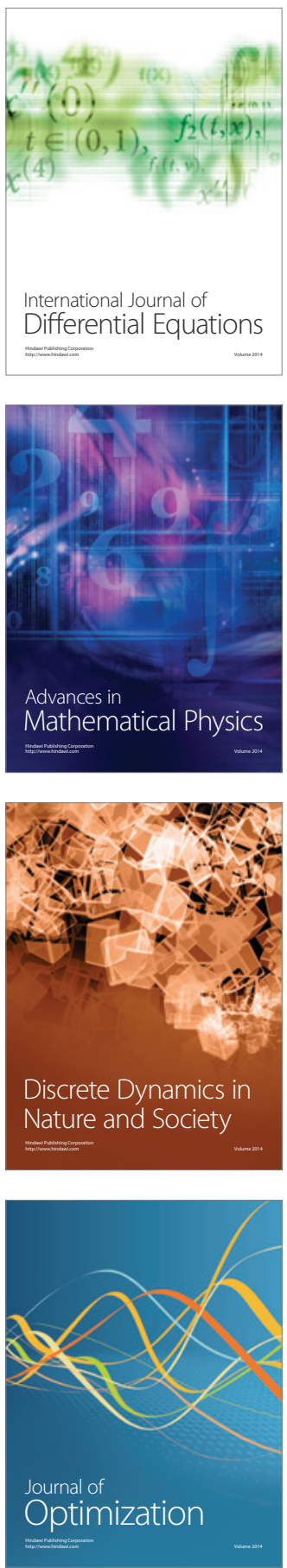